\title{
On the Use of a Dielectric Matching Layer for Ultra Wideband Medical Applications
}

\author{
Ali Khaleghi ${ }^{1,4}$, Raúl Chávez-Santiago ${ }^{1,2,3}$, llangko Balasingham ${ }^{1,2,3}$ \\ ${ }^{1}$ The Intervention Center, Oslo University Hospital \\ ${ }^{2}$ Institute of Clinical Medicine, University of Oslo \\ ${ }^{3}$ Norwegian University of Science and Technology (NTNU) \\ ${ }^{4} \mathrm{~K}$. N. Toosi University of Technology (KNTU) \\ +(47) 23070123 \\ E-mail: ali.khaleghi@rr-research.no
}

\begin{abstract}
Ultra wideband (UWB) radio signals have gained significant attention for emerging medical applications. Communication with medical implanted sensors and actuators, biomedical radar, microwave imaging, and microwave hyperthermia are some examples of said applications. All these diagnostic and therapeutic methods necessitate the propagation of electromagnetic (EM) waves toward or from the human body. In such scenarios, a segment of the wave propagation path is inside the human body and the other segment is outside, i.e., in the free-space. This geometry creates an impedance mismatch between the two propagation environments, which results in inefficient power transmission of the EM wave. In order to improve the EM wave coupling to/from the body, a matching layer of dielectric material with the appropriate permittivity value can be used. This paper analyzes the effect of a single layer of dielectric material on the propagation of a UWB wave through the human chest in the 100$1000 \mathrm{MHz}$ frequency range. Path loss models for different matching layer materials were obtained and a guideline for selecting the appropriate permittivity value is presented.
\end{abstract}

\section{Categories and Subject Descriptors}

H.4.3 [Information Systems Applications]: Communications Applications - computer conferencing, teleconferencing, and videoconferencing.

\section{General Terms}

Design, Performance, Reliability, Verification.

\section{Keywords}

Dielectric materials, matching layer, medical applications, radio propagation, ultra wideband.

\footnotetext{
Permission to make digital or hard copies of all or part of this work for personal or classroom use is granted without fee provided that copies are not made or distributed for profit or commercial advantage and that copies bear this notice and the full citation on the first page. To copy otherwise, or republish, to post on servers or to redistribute to lists, requires prior specific permission and/or a fee.

Bodynets'12, September 24-26, 2012, Oslo, Norway.

Copyright 2010 ACM 1-58113-000-0/00/0010 ..\$10.00.
}

\section{INTRODUCTION}

Radio microwaves are used in a number of medical applications such as the communication interface for implanted medical sensors and actuators, medical radar, medical imaging, hyperthermia, etc. All these diagnostic and therapeutic methods necessitate the propagation of electromagnetic waves toward or from the human body. In such scenarios, a segment of the wave propagation path is inside the human body and the other segment lies in the free-space. Due to the significant impedance differences between the two propagation environments, namely human tissues and air, the power transmission of the electromagnetic (EM) wave is rather inefficient. In order to improve the EM wave coupling to/from the body, a layer of dielectric material with the appropriate permittivity can be applied on the skin of the patient; this dielectric material is referred to as a dielectric matching layer (or simply a matching layer). This procedure is analogous to ultrasonic transducers for medical imaging, in which the use of one or more layers of matching material on the patient's skin improves the efficiency and bandwidth of the device [1]. Similarly, the use of a dielectric matching layer for radio waves in biomedical applications can increase the depth the radio wave penetrates inside the human body. For instance, the use of a matching layer in radiometry and hyperthermia can improve the monitoring and focusing of signals for the target location inside the body [2]-[4]. Likewise, a matching layer can improve the image quality in microwave imaging [5]. Most of these applications of radio waves in medicine use narrowband (NB) signals. In this case, the frequency-dependent dielectric properties of the human tissues can be ignored and determining the appropriate permittivity for the material of the matching layer is relatively simple.

However, ultra wideband (UWB) technology has gained significant attention for the aforementioned biomedical applications in the last years. One of the biggest advantages of UWB with respect to NB is the high temporal resolution of the signals, which adds significant improvement to the system performance. For instance, UWB signals can provide tighter focusing on small areas for microwave hyperthermia [6]. In the same vein, the UWB medical radar can easily distinguish the different layers of tissues inside the body [7], thereby improving the quality of the images obtained through it. It has also been demonstrated in [8] that the use of UWB for communications with a capsule endoscope can provide high data rate transmissions, 
which might enable the use of high definition (HD) video in real time. Nevertheless, recent studies of UWB signals propagating through human tissues have demonstrated that significant attenuation is expected as the depth increases [9]. Moreover, the material properties of the human tissues vary significantly with frequency; this fact cannot be neglected when using UWB signals. Hence, the human body behaves as a frequency-dependent dispersive propagation environment to UWB signals. This attenuation problem can be alleviated by using a dielectric matching layer. In a simple manner, it is expected that the matching layer increases the UWB signal strength for a given depth. However, due to the frequency-dependent dispersive propagation environment, the path loss scattering and the efficiency of the signal bandwidth can be affected; these effects have not been investigated in the literature.

Therefore, this paper presents a study of the dielectric matching layer for UWB signals propagating from the free-space into the human body. For this sake, a heterogeneous model of the human body was used for numerical EM computations. This anatomical model includes the frequency-dependent dielectric properties of the different human tissues by using the Cole-Cole model within 100-1000 MHz; this frequency band has big potential for UWB medical applications [10]. For simplicity, a plane wave scenario was simulated with a single layer lossless dielectric boundary condition applied on the chest area. Different materials were considered, the permittivity of which varied from $\varepsilon_{r}=1$ for the air to $\varepsilon_{r}=70$ for a highly dense dielectric material. The power of the propagating UWB signal was evaluated for various depths inside the chest and a path loss model was obtained for each of the different matching layers considered in the simulations. The implications of these results for selecting the appropriate dielectric material for the matching layer are thoroughly discussed.

The rest of the paper is organized as follows: Section 2 describes in detail the simulation scenario used in this study. Section 3 presents the results and a guideline for selecting the appropriate matching layer. Finally, Section 4 summarizes our conclusions.

\section{SIMULATION SCENARIO}

The simulation setup based on a heterogeneous anatomical model is illustrated in Figure 1. This anatomical model is based on data from the Visual Human Project ${ }^{\circledR}$ of the National Laboratory of Medicine (NLM) [11]. In this model, the human body is represented by voxels with a spatial resolution of $1 \mathrm{~mm}$. This resolution allows for the accurate representation of the shape of most of the internal organs. To use this model in the EM simulations, the voxels were classified according to their different dielectric material properties. Thirty-two different tissues with their respective dielectric parameters, conductivity and permittivity, were defined. Although the whole body can be used for the numerical computations required for this study, only the human torso was considered in our simulations. This was done in order to reduce the simulation time and memory consumption for the EM computations. In addition, our study considered wave propagation inside the chest only; hence, using the torso is an appropriate selection that prevents a replica of the wave to penetrate from the cut area of the model that might alter the received power calculations.

The body was exposed to an incoming plane wave from the front direction. Both electric and magnetic field probes were placed inside the chest arranged in a rectangular cube of $140 \mathrm{~mm}(w-$ axis), $160 \mathrm{~mm}$ ( $u$-axis), and $80 \mathrm{~mm}$ ( $v$-axis). The probes were separated from each other a distance of $20 \mathrm{~mm}, 10 \mathrm{~mm}$, and 20 $\mathrm{mm}$ in each axis, respectively. The total number of probes was 680 with 85 probes on each plane parallel to the skin ( $u v$-plane). Due to the curvature of the torso, the probes had different distance to the chest surface (skin). The average depth from the skin to the first plane of probes was $20 \mathrm{~mm}$. The probes were embedded in different tissues such as blood, heart, bones, cartilages, fat, lung, and muscles. The probes were frequency-independent isotropic radiating antennas with a defined polarization. It was assumed that the probe arrays did not have any mutual coupling. The electric-field probes were considered to be in co-polar mode with the polarization of the incoming wave, i.e., in the propagation direction into the body, whereas the magnetic-field probes were orthogonal to the electric ones. The boundary conditions for the torso were considered as perfect matching layer (PML), thus the wave reflections from the environment were ignored.

The EM simulation software used in our study was CST Microwave Studio ${ }^{\circledR}$, which applies the Finite Integration Technique (FIT) to solve the integral form of the Maxwell's equations in the time-domain. This tool allows importing arbitrary voxel data sets. Hence, incorporating the anatomical model into this $3 \mathrm{D}$ simulation environment was straightforward.

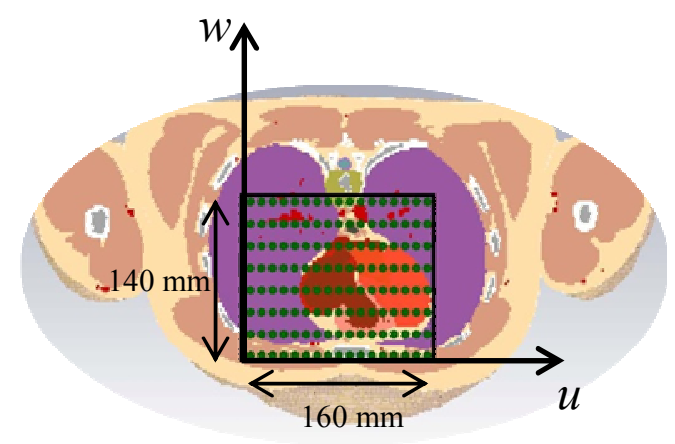

(a)

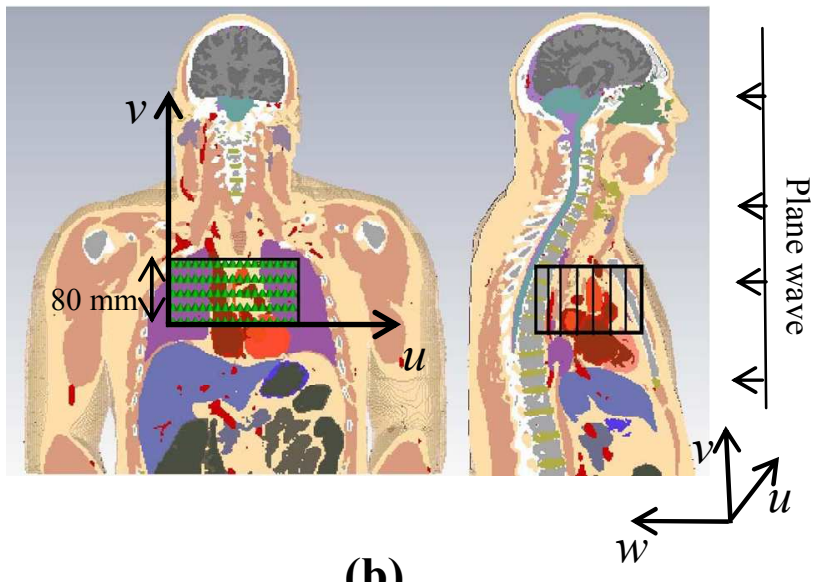

(b)

Figure 1. Simulation model of the human torso; (a) top view, and (b) vertical front (left) and side (right) views. 


\subsection{Dielectric Properties of Human Tissues}

The frequency-dependent dielectric properties of the human tissues, permittivity and conductivity, were provided by Gabriel [12] based on the Cole-Cole model. However, incorporating the high complex material properties in the numerical simulations demands extremely long computational time [13]. Our own investigations [14], [15] revealed that a simplified equation for describing the frequency-dependent complex permittivity of the human tissues can be obtained by using a second-order polynomial, which fits accurately Gabriel's data. This is given as

$$
\varepsilon_{r}(\omega)=\varepsilon_{r}^{\prime}-j \varepsilon_{r}^{\prime \prime}=\varepsilon_{\infty}+\frac{\beta_{0}+j \omega \beta_{1}}{\alpha_{0}+j \omega \alpha_{1}-\omega^{2}}
$$

where $\varepsilon_{r}^{\prime}$ and $\varepsilon_{r}^{\prime \prime}$ are defined as the real and the imaginary parts of the complex permittivity, $\varepsilon_{r}(\omega)$, respectively, $\omega$ is the angular frequency, and $\varepsilon_{\infty}$ is the permittivity at infinite frequency. $\alpha_{0}, \beta_{0}$, $\alpha_{1}, \beta_{1}$ are fitting parameters that were estimated for different tissue materials by fitting to Gabriel's four pole Cole-Cole data using Newton method and least square fitting. We have obtained the fitting parameters for the tissue materials found in the torso, namely blood, aorta, blood vessels, bones, cartilage, fat, intestine, nerves, liver, lung, marrow, muscles, skin, stomach, and ventricles. The approximation in (1) was incorporated in the EM simulations. We note, however, that the approximation in (1) is valid for the selected frequency band only, i.e., $100-1000 \mathrm{MHz}$, and not to the whole range of frequencies as proposed by the Cole-Cole model. Figure 2 and 3 show the frequency variation of the conductivity and permittivity for different human tissues, respectively. As shown, the dielectric properties are considerably different for different tissues. In addition, the material properties are highly frequency-dependent. This frequency dependency can distort the shape of any transmitted pulse through the human tissues, which behave as a frequency-selective attenuating channel; UWB signals are particularly prone to suffer from this effect.

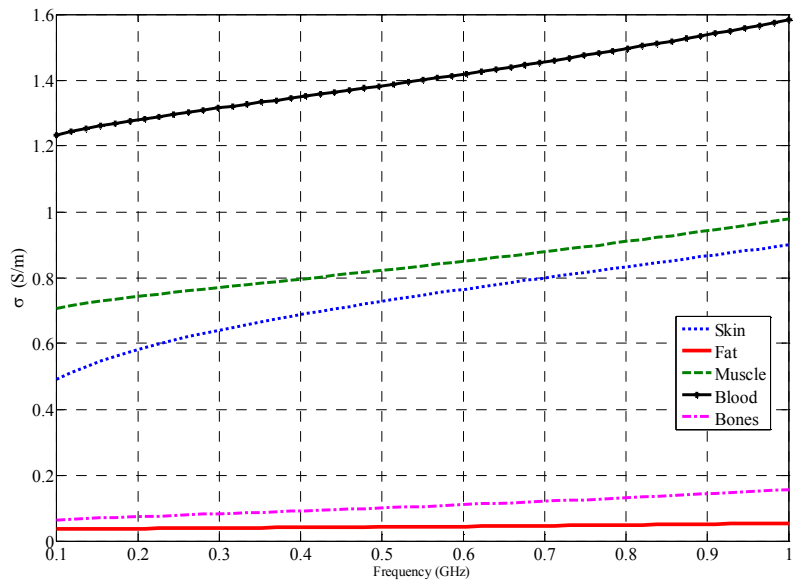

Figure 2. Conductivity of different human tissues as a function of frequency.

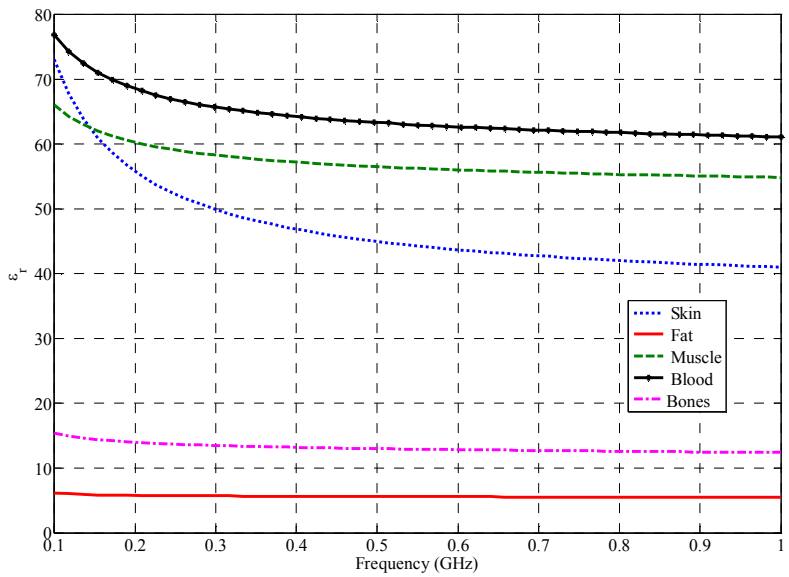

Figure 3. Permittivity of different human tissues as a function of frequency.

\subsection{Simulation of the Matching Layer}

In order to simulate the matching layer, the entire environment surrounding the torso was filled with a lossless dielectric material, i.e., the matching layer was assumed to be of infinite size. The simplest dielectric material is the air with a permittivity equal to 1 ; this case was considered as the propagation path without a matching layer. Other different permittivity values were used, namely $\varepsilon_{r}=3,5,10,20,30,40,50,60$, and 70 , for each of the different dielectric matching layer cases. The incoming plane wave was excited with a UWB Gaussian pulse with $-20 \mathrm{~dB}$ spectral bandwidth (with respect to the peak spectrum value) covering 100-1000 MHz; Figure 4 depicts the transmitted pulse shape in the time domain whereas Figure 5 shows its power spectral density. The total energy of the incoming wave for all the simulations was normalized to unity, thus on the body surface the UWB signal energy had a constant value of $0 \mathrm{~dB} \mathrm{~J} / \mathrm{m}^{2}$.

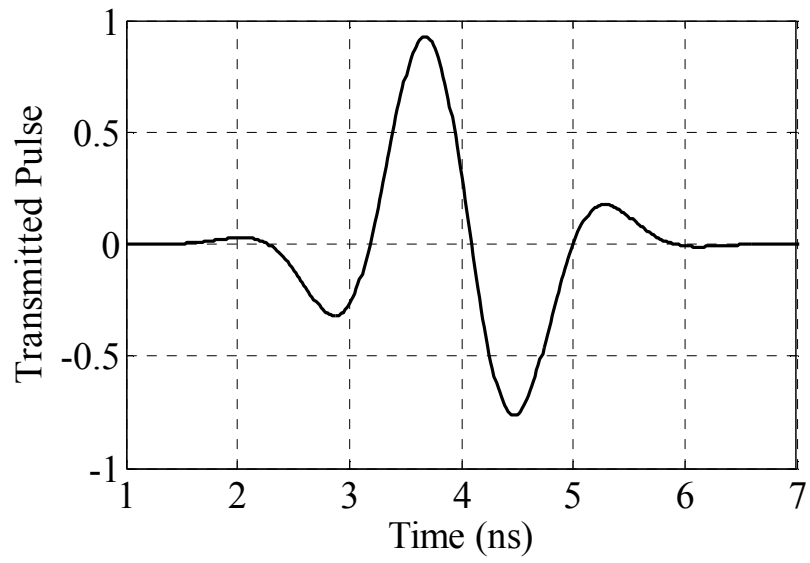

Figure 4. Ultra wideband pulse shape in the time domain. 


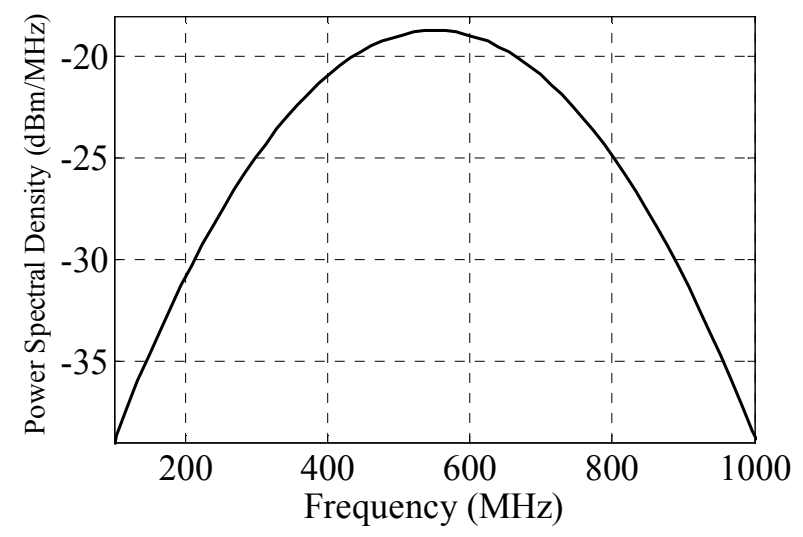

Figure 5. Power spectral density of the transmitted pulse.

\section{RESULTS}

\subsection{Path Loss without a Matching Layer}

The path loss is a measure of the average attenuation of a signal as it propagates from a radiation source to a receiving point. The total received energy at each probe was obtained by integrating the Poynting vector during an observation time interval. The Poynting vector, $S_{u, v, w}(t)$, describes both the direction of propagation and the power density of the electromagnetic wave, and can be written as

$$
S_{u, v, w}(t)=E_{u, v, w}(t) \times H_{u, v, w}(t) \quad \mathrm{W} / \mathrm{m}^{2}
$$

where $E_{u, v, w}(t)$ and $H_{u, v, w}(t)$ are the electric and magnetic field, respectively. Due to the fact that the plane wave was polarized in the $v$-axis direction, the co-polar field intensity inside the body was significantly larger compared to the cross-polar field; this was verified through simulations. Thus, only $E_{y}$ and $H_{u}$ were calculated, which means that the power flow direction was along the $w$-axis. The UWB signal energy density, which was received inside the body at different depths from the body surface, was calculated by integrating the power density over the whole observation time; this time interval was sufficiently large to include $99 \%$ of the signal energy. Hence, the signal energy is given by

$$
e_{w}=\int_{t}\left|S_{w}(t)\right| d t, \quad \tau_{0}<t<\tau_{\max } \quad \mathrm{J} / \mathrm{m}^{2}
$$

where $\tau_{0}$ and $\tau_{\max }$ delimit the time window of the received signal energy for the selected channels inside the chest. Although the probes were arranged in several planes that form a cubic shape as described previously, the contour of the chest surface was not a straight line. Therefore, the path loss at each individual probe was computed and plotted versus its actual depth from the skin. Figure 6 shows the path loss when no matching layer is applied on the torso, i.e., when the environment surrounding the torso is air with $\varepsilon_{r}=1$. A depth-dependent equation was fitted to the obtained data. A simple power law function was a good fit for the average path loss (see solid line in Figure 6). The path loss variations around the average value are known as scattering. These variations are caused by the different material properties surrounding each probe. The statistical characterization of the scattering is necessary for the calculation of the link margin. As shown, the scattering increases with depth. For simplicity, the probability density function (PDF) of the scattering (in decibels) was approximated by a single function regardless of depth. The scattering can be modeled by a Gaussian distributed random variable (RV), $\mathcal{N}$, with zero mean $\mu=0$ and standard deviation $\sigma$, i.e., $\mathcal{N}(0, \sigma)$. Hence, the expression for the propagation loss in decibels as a function of depth including the scattering term is given as

$$
L_{[\mathrm{dB}]}(d)=L_{0[\mathrm{~dB}]}+a\left(d / d_{0}\right)^{n}+\mathcal{N}(0, \sigma)
$$

where $d$ is the depth from the skin in millimeters $(1<d<100), a$ is a fitting constant with value $0.18, d_{0}$ is the reference depth (1 $\mathrm{mm}), L_{0}$ is the loss cross point $(10.6 \mathrm{~dB})$, and $n$ is a path loss exponent with value 0.93 . In this case, the scattering was modeled as $\mathcal{N}(0,4)$ as shown in Figure 7.

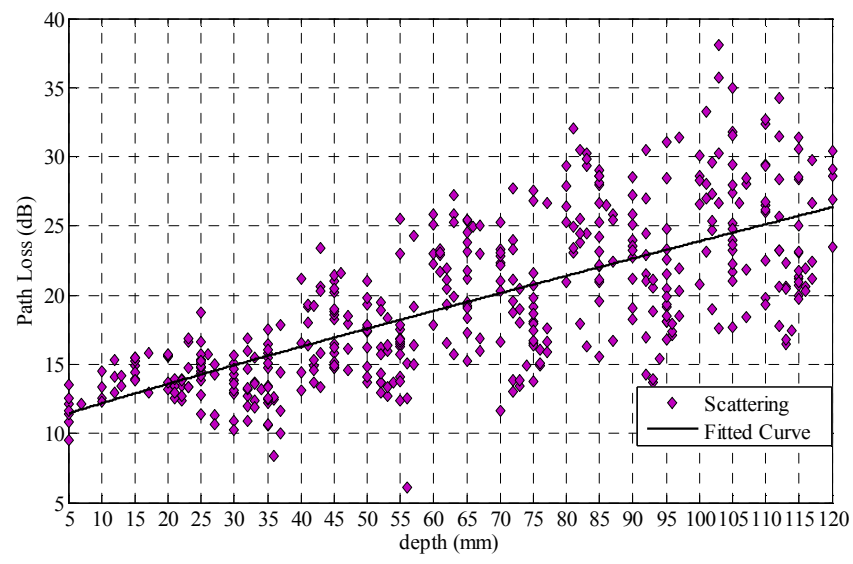

Figure 6. Path loss (dB) versus depth $(\mathrm{mm})$ without a matching layer. The scattering values are also illustrated.

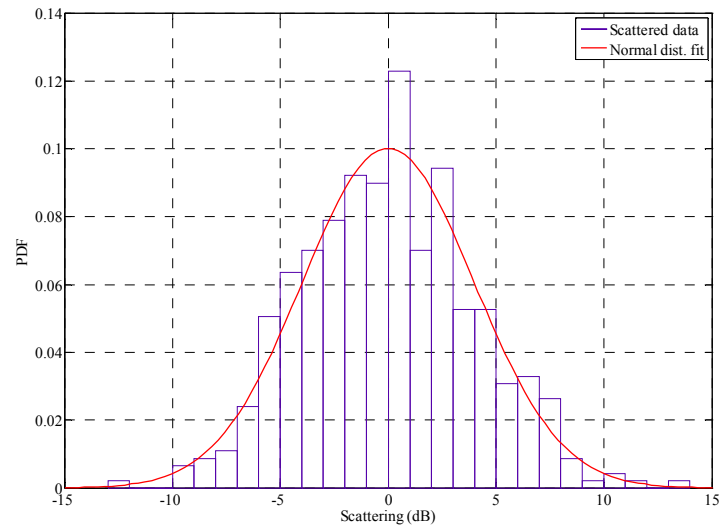

Figure 7. PDF of the scattering when no matching layer is applied on the skin. 


\subsection{Path Loss with a Matching Layer}

Similarly, the path loss for the different dielectric materials used as a matching layer was computed in each case. Figure 7 shows the fitted curves for path loss versus depth for the different dielectric materials. As seen, by increasing the permittivity of the matching layer material the path loss is significantly reduced. For instance, for $5 \mathrm{~mm}$ depth the path loss without a matching layer is $11.8 \mathrm{~dB}$, whereas this value is reduced to $8 \mathrm{~dB}$ for $\varepsilon_{r}=3$, and even further to $2.5 \mathrm{~dB}$ for $\varepsilon_{r}=20$. Beyond this value, $\varepsilon_{r}=20$, further increase of permittivity shows a saturation effect, i.e., marginal improvement is achieved with higher values of $\varepsilon_{r}$. Furthermore, the value of path loss reduction can vary with depth for the different dielectric materials. In all these cases, the path loss can still be expressed by (4); however, the parameters must be modified according to the dielectric material used. Table 1 summarizes the parameter values for path loss and scattering for the different permittivity values of the matching layer.

\subsection{Selecting the Appropriate Material}

Figure 7 can be depicted in a way that highlights the effects of permitivity. Hence, Figure 8 shows the path loss versus both depth and permitivity. In this figure one can see more clearly the saturation of the path loss as the permittivity increases. In order to provide a guideline for the selection of the appropriate permittivity of the material for a single matching layer to be used in a specific biomedical application that requires the EM waves reaching properly certain depth, we define a compression level, $P_{0}$, which is the number of decibels below the maximum path loss (saturation value) for the given depth. The compression level can be viewed as a target improvement in path loss. We considered several values for $P_{0}$, namely $P_{0}=1,2,3$ and $4 \mathrm{~dB}$; the vertical curves in Figure 7 correspond to each of these values and help to select the adequate permittivity for a given depth and compression level. For example, if a medical application requires reaching a depth of $70 \mathrm{~mm}$ and a compression level of $1 \mathrm{~dB}$ is set as a target, the dielectric material for the matching layer must have a permittivity of $\varepsilon_{r} \approx 15$. This value is obtained by tracing a horizontal line from the value of $70 \mathrm{~mm}$ in the vertical axis until it intersects the curve corresponding to $P_{0}=1 \mathrm{~dB}$; then, a vertical line is traced down from this point to the horizontal axis in order to find the adequate permittivity value. Similarly, if the compression level is set to $4 \mathrm{~dB}$, the permittivity of the material for the matching layer must have a value of $\varepsilon_{r} \approx 3.5$.

However, from a practical point of view, the permittivity obtained with the help of Figure 8 cannot always be matched with existing materials, and sometimes a compromise has to be accepted. For the sake of information, olive oil at $37^{\circ} \mathrm{C}$ has a permittivity value of $\varepsilon_{r}=3.2$, glycerol has a value of $\varepsilon_{r}=42$, and distilled water at $37{ }^{\circ} \mathrm{C}$ has a permittivity of $\varepsilon_{r} \approx 80$.

Table 1. Parameters of path loss and scattering for different permittivity values.

\begin{tabular}{|c|c|c|c|c|}
\hline $\begin{array}{c}\text { Permittivity } \\
\text { value }\end{array}$ & $a$ & $n$ & $L_{0[\mathrm{~dB}]}$ & $\sigma_{[\mathrm{dB}]}$ \\
\hline \hline 1 & 0.18 & 0.93 & 10.6 & 4 \\
\hline 3 & 0.97 & 0.60 & 5.5 & 4.3 \\
\hline 5 & 1.07 & 0.60 & 3.4 & 4.3 \\
\hline 10 & 0.95 & 0.64 & 0.9 & 4 \\
\hline 20 & 0.49 & 0.78 & 0.3 & 3.6 \\
\hline 30 & 0.33 & 0.87 & 0 & 3.4 \\
\hline 40 & 0.27 & 0.92 & 0 & 3.25 \\
\hline 50 & 0.25 & 0.94 & -0.2 & 3.2 \\
\hline 60 & 0.24 & 0.95 & -0.3 & 3.1 \\
\hline 70 & 0.23 & 0.96 & -0.3 & 3 \\
\hline
\end{tabular}

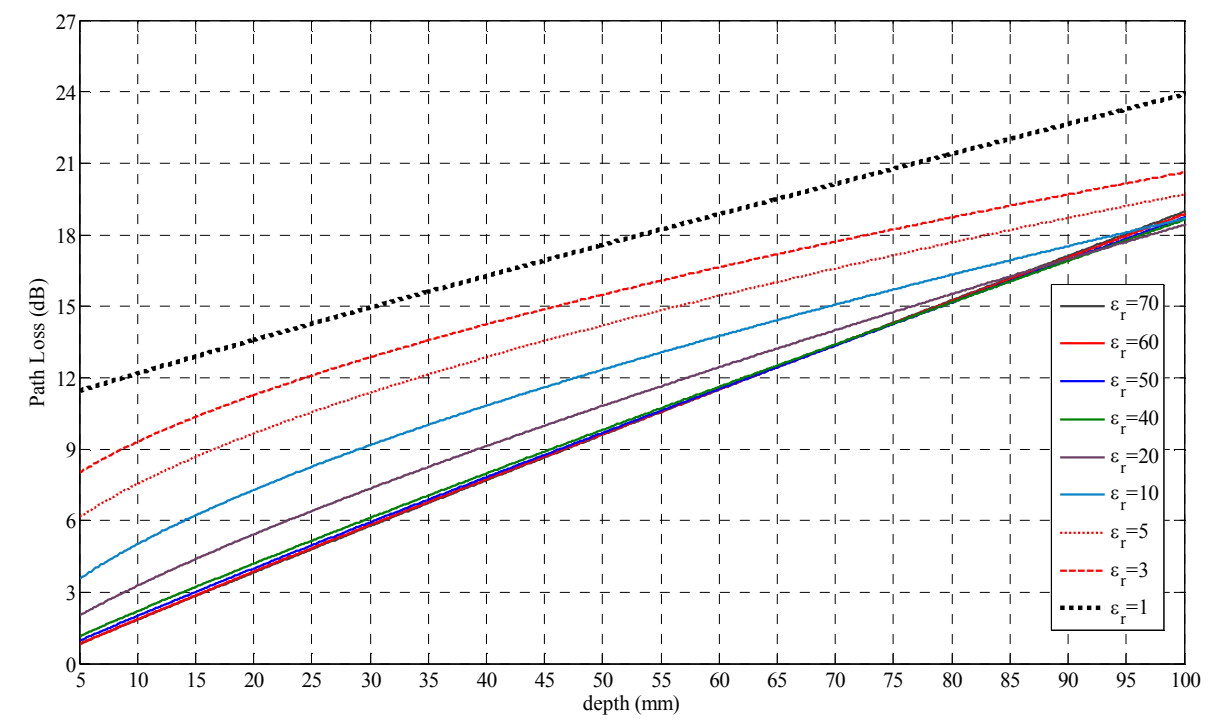

Figure 8. Path loss (dB) versus depth $(\mathrm{mm})$ for different dielectric matching layers. 


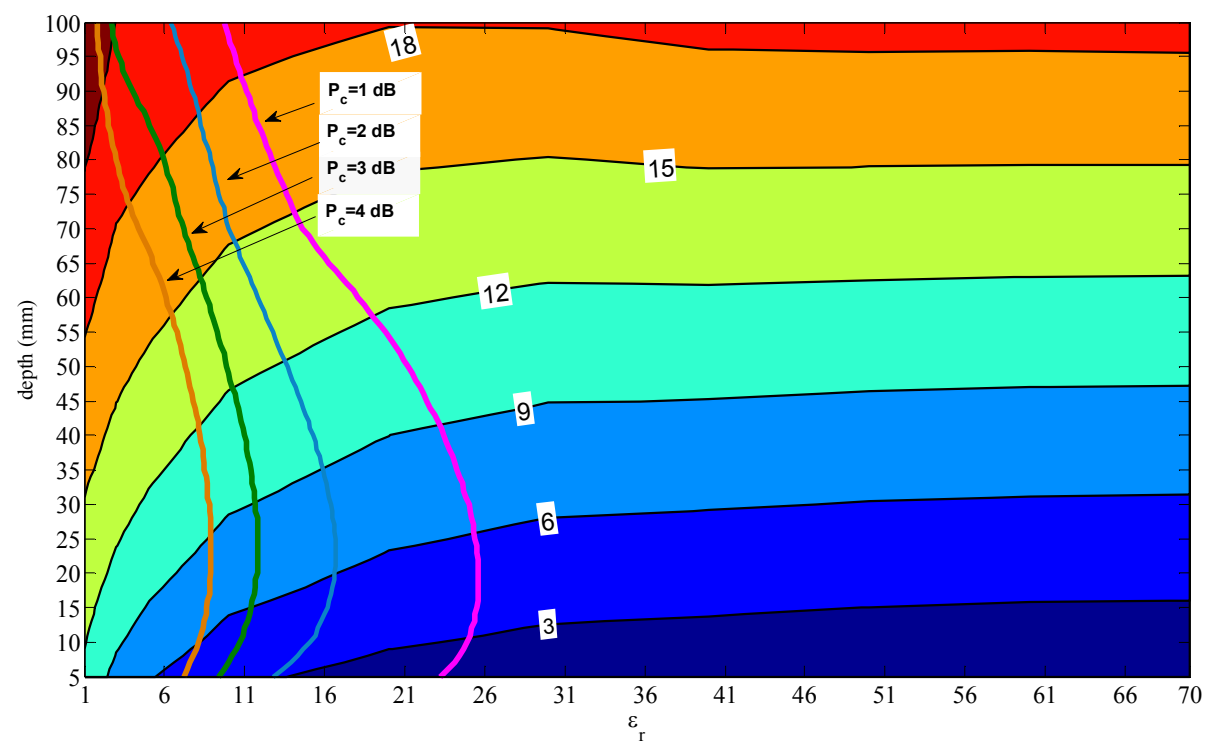

Figure 9. Path loss $(\mathrm{dB})$ versus depth $(\mathrm{mm})$ and permittivity.

\section{CONCLUSIONS}

In this paper we have investigated the effects of a dielectric matching layer applied on the human chest on the path loss suffered by an ultra wideband electromagnetic wave propagating from the outer to the inner body. Our simulations have evidenced that a matching layer of dielectric material with a permittivity value larger than 1 reduces the propagation loss suffered by the electromagnetic wave. This reduction of path loss can reach up to $10 \mathrm{~dB}$, but in most cases the improvement is below this value and it depends on the depth inside the chest and the permittivity of the matching layer material. An interesting finding is that above a certain value of permittivity, approximately $\varepsilon_{r} \approx 20$, only a marginal reduction of the path loss is obtained with higher values. Therefore, we provided a guideline to obtain the adequate permittivity value for a target depth and for a target path loss reduction. This is an important contribution that can greatly help designers of biomedical devices that use ultra wideband signals in the $100-1000 \mathrm{MHz}$ frequency range.

The continuation of this work will focus on the effects of the matching layer on the effective bandwidth of the propagating electromagnetic wave. Our recent research has demonstrated that the frequency-dependent properties of the human tissues impact negatively on the effective bandwidth of the system, i.e., the effective bandwidth of the ultra wideband signal is reduced as frequency and depth increase. We hypothesize that the use of a matching layer counters this negative effect. It is also desirable to provide a mathematical expression for the computation of the adequate permittivity value instead of the graphic we presented herein. Frequencies above $1000 \mathrm{MHz}$ have to be investigated too. Clearly, much remains to be done.

\section{ACKNOWLEDGMENTS}

This work is part of the MELODY Project, which is financed by the Research Council of Norway under the contract 187857/S10.

\section{REFERENCES}

[1] Haller, M. I., and Khuri-Yakub, B. T. 1993. Tapered acoustic matching layers. In Proceedings of IEEE Ultrasonics Symposium (1993). 505-508. DOI= 10.1109/ULTSYM.1993.339558

[2] Giamalaki, M. I., Karanasiou, I. S., and Uzunoglu, N. K. 2007. Focused microwave radiometry from a possible functional imaging perspective: theoretical optimization of the properties of a microwave radiometry system. In Proceedings of the 4th International Conference on Imaging Technologies in Biomedical Sciences, from Medical Images to Clinical Information - Bridging the Gap (Milos Island, Greece, September 22-28, 2007). DOI= http://dx.doi.org/10.1088/1748-0221/4/05/P05011

[3] Karathanasis, K. T., Gouzouasis, I. A., Karanasiou, I. S., Giamalaki, M. I., Stratakos, G., and Uzunoglu, N. K. 2010. Noninvasive focused monitoring and irradiation of head tissue phantoms at microwave frequencies. IEEE Trans. Inform. Technol. Biomed., 14 (May 2010), 657-663.

[4] Arunachalam, K., Maccarini, P. F., De Luca, V., Bardati, F., Snow, B. W., and Stauffer, P. R. 2010. Modeling the detectability of vesicoureteral reflux using microwave radiometry. Phys. Med. Biol. 55 (Sep. 2010), 5417-5435.

[5] Fear, E. C., Li, X., Hagness, S. C., and Stuchly, M. A. 2002. Confocal microwave imaging for breast cancer detection: localization of tumors in three dimensions. IEEE Trans. Biomed. Eng., 49 (Aug. 2002), 812-822.

[6] Converse, M., Bond, E. J., Van Veen, B. D., and Hagness, S. C. 2006. A computational study of ultra-wideband versus narrowband microwave hyperthermia for breast cancer treatment. IEEE Trans. Microwave Theory Tech., 54 (May 2006), 2169-2180.

[7] Jacobsen, S. and Birkelund, Y. 2010. Improved resolution and reduced clutter in ultra-wideband microwave imaging 
using cross-correlated back projection: experimental and numerical results. Intl. J. of Biomedical Imaging. 2010 (Dec. 2010). DOI= $10.1155 / 2010 / 781095$

[8] Khaleghi, A., Chávez-Santiago, R., and Balasingham, I. 2010. Ultra wideband pulse-based data communications for medical implants. IET Communications, 4 (Oct. 2010), 1189-1197.

[9] Khaleghi, A., Chávez-Santiago, R., and Balasingham, I. 2010. Ultra-wideband statistical propagation channel model for implant sensors in the human chest. IET Microwaves, Antennas \& Propagation, 5 (2011), 1805-1812.

[10] Khaleghi, A., Chávez-Santiago, R., Liang, X., Balasingham, I., Leung, V., and Ramstad, T. A. 2010. On ultra wideband channel modeling for in-body communications. In Proceeding of IEEE Intl. Symp. on Wireless Pervasive Computing (Modena, Italy, May 5-7, 2010). DOI= http://dx.doi.org/10.1109/ISWPC.2010.5483804

[11] Gjonaj, E., Bartsch, M., Clemens, M., Schupp, S., and Weiland, T. 2002. High-resolution human anatomy models for advanced electromagnetic field computations. IEEE Trans. Magn., 38 (Mar. 2002), 357-360.

[12] Gabriel, C. 1996. Compilation of the dielectric properties of body tissues at RF and microwave frequencies. Technical Report. Brooks Air Force, San Antonio, TX.

[13] Kang, K., Chu, X., Dilmaghani, R., and Ghavami, M. 2007. Low complexity Cole-Cole expression for modeling human biological tissues in (FD) ${ }^{2} / \mathrm{TD}$ method. Electronics Letters, 43 (Feb. 2007), 143-144.

[14] Khaleghi, A., and Balasingham, I. 2009. On non-line-ofsight on-body ultra wideband (1-6 GHz) channel characterization using different antenna polarizations. IET Microwaves, Antennas \& Propagation, 3 (2009) 1019-1027.

[15] Khaleghi, A., and Balasingham, I. 2009. Improving in-body ultra wideband communication using near-field coupling of the implanted antenna. Microwave and Optical Technology Letters, 51 (Mar. 2009), 585-589. 